\title{
Prospects of Electronic Systems
}

\section{Bruno Riccò* and Marco Grossi}

Department of Electrical, Electronic, and Information Engineering, University of Bologna, Italy

The field of Electronic Systems, implementing all ICT applications, experienced a big growth during the last decades, with products ever more powerful but, (at the same time, also smaller, better cheaper, safer,...). Transistors density on chips still is still increasing approximately following Moore's law [1], but it is inevitably approaching its limits, due to technical problems (excessive power consumption because of higher frequencies and transistor count, worse noise margins due to reduced supply voltages, statistical dispersion of transistor parameters,...) as well as because of economic reasons (skyrocketing cost of fab lines). Nevertheless, the current trend in microelectronics will certainly continue for at least the next decade. Thus some forecasts can be done for Electronic Systems in such a timeframe.

Starting with technology, real engine of the whole field, it is convenient to distinguish between two types of developments, generally known as "More Moore" and "More than Moore, respectively [2,3].

The former refers to the evolution of the current silicon technology towards its limits that will be inevitably reached sooner or later although it is not yet clear when this will be and what the extreme products of this process will ever be. The "More Moore" approach is inherently evolutionary, rather that revolutionary, hence, it is also "certain", as it represents the extrapolation of the path currently followed by the high-tech industry. In spite of this, however, it will require formidable $\mathrm{R} \& \mathrm{D}$ efforts in all aspects of microelectronics (fabrication processes, materials, equipment, plants, design,...).

In facts, the path to be followed has been described up to 2016 by the International Technology Roadmap for Semiconductors (ITRS) and since then by the International Roadmap for Devices and Systems (IRDS) [4], i.e., expected to continue covering (at least) the next decade.

The resulting products will be components of outstanding complexity (billions of active elements) and functionality, addressing large markets in order to justify the extremely high cost of their fabrication. To this aim, a big effort will be devoted to the architecture of real "Systems on Chip" (SoC) that, in addition to ever higher performance, will exhibit other general purpose functionalities, such as, for instance: work correctly with increasingly critical components and/ or in the presence of a number of faults (fault-tolerance); recognize the presence of fatal faults (self-checking), otherwise impossible to discover in the midst of a gigantic multitude of transistors and interconnects; (possibly) be able to repair or to reconfigure themselves (self-repairing and re-configurability, respectively) to neutralize faults and/or to adapt to new tasks.

But the development in architectures will also concern other aspects of SoC, that will become ever more "modular", to make complexity compatible with feasibility, and flexible (with the incorporation of inherently different parts, such as digital and analog circuits, power devices, sensors, actuators, switches,...).

Of course, design methodology and tools will necessarily accompany the evolution of SoC, with the need of ever higher levels of system representations.

As for applications, the new components will allow ever more powerful products featuring the convergence of computers, telecommunications and instrumentations, which, in turn, will take our current "Information Society" to new frontiers (such as, for instance, to the Internet of Things -IoT), with widespread use of Artificial Intelligence, thinking objects, robots, wearable systems, immersive experiences,....

Substantial contributions are also to be expected for packaging, playing an important role in determining dimensions, performance and costs of electronic systems. In this respect, so far the efforts have been primarily focused on better ways to interconnect chips, with or without their own packages (with solutions such as Multi-Chip-Modules and Systems-In-Packages). In the future, however, all complementary parts should be realized with thin film technology on the system (layered) mechanical substrates, together with the interconnects. The results will be System-on-Package (SOP), much smaller than the present ones (for the same functionality), exhibiting higher performance and lower (unitary) costs.

These types of packages will be able to accommodate components realized with different technologies and materials (bare or packaged digital circuits, RF amplifiers and oscillators, antennas, capacitors, inductors, resistors, filters, crystals, and waveguides but also sensors, nanotubes, nanobelts, MEMS). In this way, each part of the system could be realized in parallel and with its optimal technology, since the remaining problems of "integration" would be handled at package level.

The use of parts realized with different technologies, make us enter the "More than Moore" field.

This term indicates two main types of development, namely: a) replacements of silicon technology when it will come to an end, probably because no longer economically convenient; b) search for alternative and/or complementary technologies able to improve the performance at system level and/or to open new fields of applications.

A key factor for this "More than Moore" approach is Heterogeneous Integration, than can be pursued using different types of substrates, including organic and flexible ones, as well as silicon wafers (containing interconnects) on which bare (flip) chips are bonded with the possibility of some sort of "3D integrations" (i.e., device stacking).

But there are also other important needs requiring ad-hoc solutions, such as, for instance, bio-chips to detect and recognize biomolecules [5], micro-systems realized in the form of capsules to travel within the human body in order to collect and transmit information

*Corresponding author: Bruno Riccò, Department of Electrical, Electronic and Information Engineering, University of Bologna, Italy, Tel: +390512093018; Fax: +390512093073; E-mail: bruno.ricco@unibo.it

Received August 26, 2017; Accepted September 15, 2017; Published September 22, 2017

Citation: Riccò B, Grossi M (2017) Prospects of Electronic Systems. J Electr Electron Syst 6: 239. doi: 10.4172/2332-0796.1000239

Copyright: ( 2017 Riccò B, et al. This is an open-access article distributed under the terms of the Creative Commons Attribution License, which permits unrestricted use, distribution, and reproduction in any medium, provided the original author and source are credited. 
(pictures and video) [6], micro-dispensers to reside within the human body and featuring sensors, actuators and processors.

Particularly, though not exclusively, in this type of applications, power independence, obtained through suitable Energy Harvesting devices [7], will play an important role.

As already mentioned, a second interest of the "More than Moore" approach is the search for replacements of silicon transistors and electronics, starting from near and interesting fields, such as optics (i.e., devices and interconnects for fully optical systems, since optic interconnects alone are already considered to connect silicon chips), but expanding also to more exotic and hypothetical ones. Among these, for instance, nano-technology, quantum electronics (quantum dots and wires, single electron transistors) and molecular electronics (i.e., molecular building blocks for the fabrication of electronic components).

Interesting, although still far away, are also suggestions such as Quantum Computing, possibly able to multiply computation power (for equivalent hardware) or the attempt to (somewhat) copy the brain architecture and operation.

Among all the possibilities mentioned above, and many others, the future with its ever accelerating pace will certainly decide which way to go. In any case, the future products of the "More Moore" approach (integrated systems featuring billions of nano-transistors) are a certainty and will be extremely hard to compete with.

\section{References}

1. Moore G (1965) Cramming More Components onto Integrated Circuits. Electronics Magazine.

2. Heinig A, Dietrich M, Herkersdorf A, Miller F, Wild T, et al. (2014) System integration-the bridge between More than Moore and More Moore. Proceedings of the conference on Design, Automation \& Test in Europe. European Design and Automation Association.

3. Bennett HS, Hutcheson GD (2011) More than Moore or More Moore: a SWOT analysis. In AIP Conference Proceedings.

4. International Roadmap for Devices and Systems (IRDS). http://irds.ieee.org/

5. Ba D, Wang D, Liu K, Hao M, Du G, et al. (2016) Nanofluidic Chips for BioMolecules Manipulation Controlled by Back Electrodes Enclosed with Glass and Polydimethylsiloxane. Journal of Computational and Theoretical Nanoscience 13: 2237-2244.

6. Ciuti G, Caliò R, Camboni D, Neri L, Bianchi F, et al. (2016) Frontiers of robotic endoscopic capsules: a review. Journal of Micro-Bio Robotics 11: 1-18.

7. Priya S, Inman DJ (2009) Energy harvesting technologies. Springer, New York. 